\title{
Seroprevalence of Fasciola gigantica infection in bovines using cysteine proteinase dot enzyme-linked immunosorbent assay
}

\author{
Niranjan Kumar, Anju Varghese and J. B. Solanki \\ Department of Parasitology, College of Veterinary Science and Animal Husbandry, Navsari Agricultural University, \\ Navsari - 396 450, Gujarat, India. \\ Corresponding author: Niranjan Kumar, e-mail: niruvet@gmail.com \\ Co-authors: AV: dranjuvarghese@gmail.com, JBS: drjbsolankivet@gmail.com \\ Received: 23-06-2017, Accepted: 11-09-2017, Published online: 06-10-2017
}

doi: 10.14202/vetworld.2017.1189-1193 How to cite this article: Kumar N, Varghese A, Solanki JB (2017) Seroprevalence of Fasciola gigantica infection in bovines using cysteine proteinase dot enzyme-linked immunosorbent assay, Veterinary World, 10(10): 1189-1193.

\begin{abstract}
Aim: The objective of the present study was to know the seroprevalence status of Fasciola gigantica infection in cattle and buffaloes using cysteine proteinase (CP) antigen in dot enzyme-linked immunosorbent assay (ELISA) format under field conditions.

Materials and Methods: As per the standard protocol, the sera were collected from the blood of 112 cattle and 38 buffaloes of coastal areas of Navsari district, South Gujarat, India. The indirect ELISA was performed on the strip of nitrocellulose paper blotted with $1 \mu \mathrm{l}$ of $\mathrm{CP}$ antigen, to detect $F$. gigantica seropositive animals.

Results: The native CP of $F$. gigantica revealed a single visible band on $10 \%$ sodium dodecyl sulfate-polyacrylamide gel electrophoresis. There was no any noted cross-reaction between the selected antigen and sera of Gastrothylax crumenifer-infected animals in ELISA. Out of 150 screened bovines, the sera of $47(31.33 \%)$ were found to be reactive in dot-ELISA, with a prevalence rate of $31.25 \%$ and $31.58 \%$ in cattle and buffaloes, respectively. The seropositive bovines with heavy, moderate, and light level of infection were $44.68 \%, 34.04 \%$, and $21.28 \%$, respectively ( $<<0.05$ between heavy and light; $\mathrm{p}>0.05$ between moderate and heavy or light). The share of $F$. gigantica seropositive and negative animals was $31 \%$ and $69 \%$, respectively. The optical density at $450 \mathrm{~nm}$ of pooled sera of seropositive bovines with heavy, moderate, and light reactivity in plate-ELISA was significantly higher with field or reference negative sera.
\end{abstract}

Conclusion: The CP-based dot-ELISA can be useful for field veterinarians for quick and timely isolation of the animals requiring urgent flukicide therapy.

Keywords: bovines, cysteine proteinase, dot enzyme-linked immunosorbent assay, Fasciola gigantica.

\section{Introduction}

Fasciolosis, the snail-borne disease, is widely prevalent in most geographical regions of the world. The infection rate caused by tropical fluke, Fasciola gigantica, varies between 10 to $80 \%$ among buffalo and cattle (as primary hosts), followed by sheep and goat [1-3]. The disease is recognized as one of the most economically important helminth infection of the ruminants [4], with worldwide monetary losses conservatively estimated at over US $\$ 3.2$ billion per annum [5]. The economic losses are due to mortality, morbidity, poor growth, and productivity loss in the infected animals [6], and hence, the prevention and control of fasciolosis could contribute significantly to improve animal production [7].

Copyright: Kumar, et al. Open Access. This article is distributed under the terms of the Creative Commons Attribution 4.0 International License (http://creativecommons.org/licenses/by/4.0/), which permits unrestricted use, distribution, and reproduction in any medium, provided you give appropriate credit to the original author(s) and the source, provide a link to the Creative Commons license, and indicate if changes were made. The Creative Commons Public Domain Dedication waiver (http://creativecommons.org/ publicdomain/zero/1.0/) applies to the data made available in this article, unless otherwise stated.
The feasible approaches to prevent and control the fasciolosis are based on reducing the population of the snail intermediate host or its timely identification followed by drug dosing in the definitive host [8]. Diagnosis of animal fasciolosis largely based on the microscopic demonstration of the parasite egg in the feces on 12-14 weeks postinfection (WPI), i.e., only possible in patent/late phase of infection [9]. Furthermore, the low sensitivity of the coprological methods resulted in the underdiagnosis of weak infection, and thus, it is not suitable for analyzing the disease condition in the large herds [10]. The pathogen causes major harm to the definitive host during its prepatent/ early phase of infection [11]. Therefore, diagnosis in the prepatent/early phase of infection is the major objective to ensure sustainability in animal production.

Serological assays are the only options for the detection of infection at an early prepatent stage using parasite-specific antigenic moieties [12]. However, the helminths are a complex organism and are known to share antigenic epitopes, resulting in cross-reaction and false-positive case [13]. Therefore, to develop a serological assay for the diagnosis of fasciolosis in the 
ruminants, continuous efforts are needed to identify and characterize the antigen for their utilization under field conditions for the early and specific detection of the infection [14-18]. Furthermore, specific diagnosis will help in the selection of a specific drug for the treatment and control of the disease, and thus, minimizes drug abuse, often leading into the development of resistant strains of the parasites [19].

An attempt to develop $F$. gigantica-specific diagnostic kit for the bovine, the Helminthology Laboratory, Division of Parasitology, Indian Veterinary Research Institute, Izatnagar - 243122 had isolated and characterized the $28 \mathrm{kDa}$ cysteine proteinase (CP) from the excretory-secretory product of the fluke [16]. This antigen-based enzyme-linked immunosorbent assay (ELISA) successfully detected fasciolosis in the buffaloes with a high level of sensitivity and specificity under field situations $[12,17,20]$. The present study aimed to know the seroprevalence status of $F$. gigantica infection in the bovines using $\mathrm{CP}$ antigen in dotELISA format under field conditions of heavy rained areas of South Gujarat of Navsari district, India.

\section{Materials and Methods}

\section{Ethical approval}

As the study was conducted with the clinical cases, so ethical committee approval was not required.

\section{Study area}

The villages of Navsari $\left(20.95^{\circ} \mathrm{N}, 72.93^{\circ} \mathrm{E}\right)$ district of Gujarat state of India are characterized with average monthly rainfall of 0 to $1663.77 \pm 448.00 \mathrm{~mm}$ in winter/summer to rainy season and the relative humidity from $29.2 \pm 4.25$ to $84.73 \pm 3.44 \%$ in February to August.

\section{Biological sample collection and processing}

Each $5 \mathrm{ml}$ of blood was collected from the jugular vein of properly restrained 112 cattle and 38 buffaloes, presented before the clinician for disease diagnosis/treatment in the clinical camps organized in the above mentioned areas, using sterile needle and syringe. The collected blood samples were allowed to coagulate and further centrifuged to obtain the sera and stored at $-20^{\circ} \mathrm{C}$ until further analysis.

\section{Reference sera and CP antigen for ELISA}

Five positive/negative reference sera and diagnostic antigen, $\mathrm{CP}$ of $F$. gigantica infection were provided by the Helminthology Laboratory, Division of Parasitology, IVRI, Izatnagar. The negative control sera were originated from the buffalo calves reared under experimental conditions for 1 year, properly dewormed, and proven as free from $F$. gigantica infection by the expert. Simultaneously, the $F$. gigantica free buffalo calves were infected with the viable metacercariae and bled at $8^{\text {th }}$ week postinfection to isolate positive control sera. The $\mathrm{CP}$ antigen was purified by the said laboratory from the in vitro released $F$. gigantica excretory-secretory products by twostep alcohol fractionation, followed by ion-exchange chromatography [16]. The integrity of the diagnostic $F$. gigantica-specific CP antigen was checked on $10 \%$ sodium dodecyl sulfate-polyacrylamide gel electrophoresis (SDS-PAGE) [16]. The said laboratory had also provided each five $F$. gigantica-positive/negative and Gastrothylax crumenifer-positive/negative buffalo's field sera, proven by postmortem followed by a fecal examination. The reactivity pattern of these sera was used to compare the positive and negative animals in ELISA format with CP antigen.

\section{Seroprevalence study}

The indirect dot-ELISA was performed as per the method described by Sriveny et al. [17] with adjustable modifications. Briefly, $\mathrm{CP}$ antigen of $1 \mu \mathrm{l}$ ( $\sim 200 \mathrm{ng}$ ) was blotted onto the rectangular strips of nitrocellulose paper (Biorad, California, USA). The antigen blotted spots were air-dried, incubated at $37^{\circ} \mathrm{C}$ for $1 \mathrm{~h}$, and stored at room temperature until required. The strips were thoroughly washed 3 times in phosphate buffer saline (PBS)-Tween-20 $(0.05 \%, 50 \mu 1$ of Tween-20 in $100 \mathrm{ml}$ of PBS of pH 7.2) wash buffer. The strips were blocked in a solution containing 5\% skimmed milk powder in PBS for $2 \mathrm{~h}$ at room temperature. The strips were thoroughly washed 3 times with wash buffer, and then, incubated in 1:50 diluted sera in $1 \%$ skimmed milk in PBS separately for $1 \mathrm{~h}$ at room temperature. After three washings, the strips were dipped in rabbit anti-bovine immunoglobulin $\mathrm{G}$ ( $\mathrm{IgG}$ )-horse radish peroxidase (HRPO) conjugate (Santa Cruz, USA) at 1:2000 dilution in 1\% skimmed milk in PBS for $1 \mathrm{~h}$ at room temperature. Following five washes, the strips were incubated at room temperature in the dark in diaminobenzidine (Sigma Chemical Company, USA) chromogenic substrate buffer $(8 \mathrm{mg}$ diaminobenzidine, $10 \mathrm{ml}$ PBS, $10 \mu \mathrm{l}$ $\mathrm{H}_{2} \mathrm{O}_{2}$, for a total $10 \mathrm{ml}$ substrate volume) for color development. The strips were thoroughly washed after visible dot formation in the several changes of the distilled water to stop further chromogenic reactions and dried on the filter paper. The positive sera of F. gigantica infection were characterized by the formation of distinct brown color dots on the paper while negative sera showed no dots. Depending on the intensity of the dot, the bovines were grouped into heavily, moderately, and lightly infected with $F$. gigantica. The sera were pooled accordingly to form heavily, moderately, lightly, and negatively $F$. gigantica-infected one, and further, analyzed in the indirect plate-ELISA.

Indirect plate-ELISA was performed as per the method described by Kumar et al. [14,15] with some modifications. The flat-bottom polystyrene microtiter plates (Greiner, Germany) were incubated overnight at $4^{\circ} \mathrm{C}$ with $100 \mu \mathrm{l} /$ well of carbonate and bicarbonate coating buffer containing $5.0 \mu \mathrm{g} / \mathrm{ml}$ of CP antigen. The wells were washed twice for 5 min with washing buffer and incubated at $37^{\circ} \mathrm{C}$ for $2 \mathrm{~h}$ with $300 \mu 1 /$ well of blocking solution, $3 \%$ skimmed milk in PBS. After thrice washings of the plate in the washing buffer, each well was loaded with $100 \mu 1$ of 1:100 diluted 
pooled sera in $1 \%$ skimmed milk prepared in PBS and incubated at $37^{\circ} \mathrm{C}$ for $1 \mathrm{~h}$. Wells were washed again 3 times with wash buffer, and $100 \mu 1$ of rabbit anti-bovine IgG-HRPO (Santa Cruz, USA) conjugate in 1:5000 dilution prepared in 1\% skimmed milk in PBS was added to each well and incubated at $37^{\circ} \mathrm{C}$ for $1 \mathrm{~h}$. After five vigorous washings, $100 \mu \mathrm{l}$ of chromogenic substrate with O-phenylenediamine dihydrochloride (OPD) (Sigma, USA) solution (OPD $-0.8 \mathrm{mg} / \mathrm{ml}$ phosphate-citrate buffer $\mathrm{pH} 5.0$ and $1 \mu \mathrm{H}_{2} \mathrm{O}_{2} / \mathrm{ml}$ substrate buffer) was added to each well and kept in the dark for color development. The reaction was stopped after the development of appropriate chromogenic reaction using $50 \mu 13 \mathrm{~N} \mathrm{HCl} /$ well. The absorbance readings were taken at $450 \mathrm{~nm}$ on an ELISA reader (Biorad, USA). The data expressed as the mean of the optical density (OD) were recorded for the grouped samples.

\section{Statistical analysis}

The results were compiled systematically, and data were analyzed using IBM SPSS Statistics 20.00 for Windows (SPSS Inc., Chicago, USA) to perform one-way ANOVA using Duncan for determination of statistical significance. The $p>0.05$ was considered as statistically non-significant.

\section{Results}

The integrity of the procured native $\mathrm{CP}$ of $F$. gigantica revealed a single observable band on the $10 \%$ SDS-PAGE, which was used to determine the anti- $F$. gigantica antibody in the cattle and buffaloes. There was no cross-reaction of the $\mathrm{CP}$ antigen with $G$. crumenifer field sera at any point of time in plate-/dot-ELISA. Out of 150 screened bovine, sera of 47 animals were found to be reactive in dot-ELISA with 31.25 and $31.58 \%$ prevalence rate in cattle and buffaloes, respectively (Figure-1). The $F$. gigantica seropositive bovines with heavy, moderate, and light level of infection were $44.68 \%, 34.04 \%$, and $21.28 \%$, respectively $(p<0.05$ between heavy and light; $p>0.05$ between moderate and heavy/light) (Figure-2). The $\%$ distribution of $F$. gigantica seropositive/negative bovines was depicted in Figure-3. The OD at $450 \mathrm{~nm}$ of pooled sera of seropositive cattle with heavy, moderate, and light reactivity in plate-ELISA was 3.2845 , 2.7135 , and 1.7225 , respectively, which was significantly higher with field negative sera (Figure-4). Likewise, OD at $450 \mathrm{~nm}$ of pooled sera of seropositive buffaloes with heavy, moderate, and light reactivity in plate-ELISA was $3.26375,2.18375$, and 1.433 , respectively, which was significantly higher with reference/field negative sera (Figure-4).

\section{Discussion}

The field applicability of the diagnostic assay is the final goal and worthwhile. Several immunodiagnostic assays were tried for the prepatent detection of fasciolosis in field setup, namely, double immunodiffusion [21], skin hypersensitivity reactions [22],

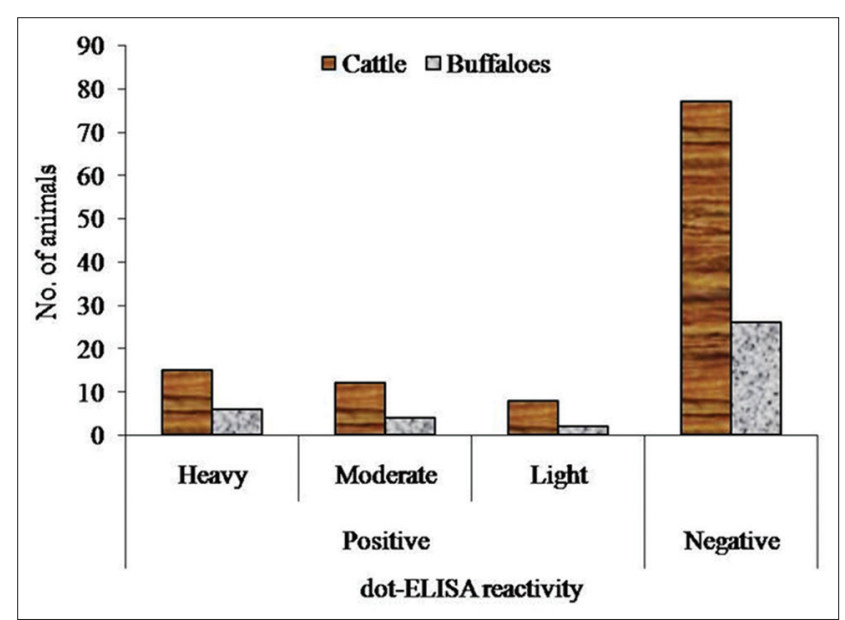

Figure-1: Dot enzyme-linked immunosorbent assay reactivity pattern of Fasciola gigantica infection.

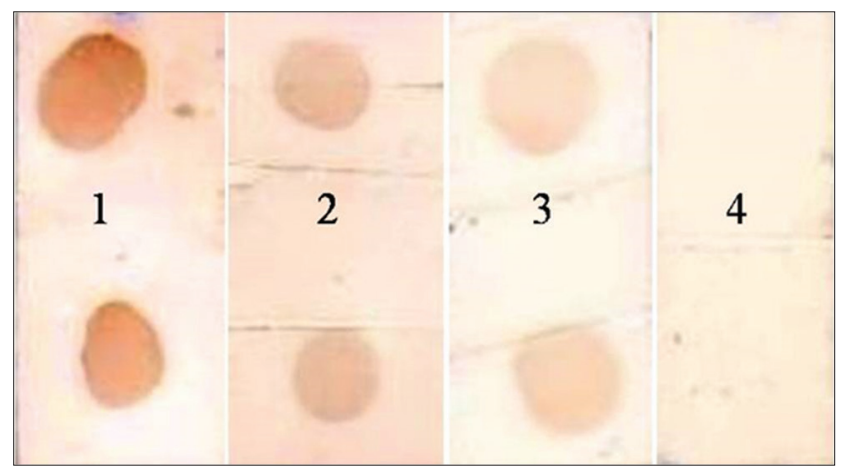

Figure-2: Representative dot strip no. 1-3 (1 - heavy, 2 - moderate, and 3 - light) and 4 showing positive and negative reactivity of Fasciola gigantica infection, respectively.

dot-ELISA [23], and dot immunoperoxidase [24] with their different acceptability levels. The dot-ELISA is more widely accepted, since the test is easy to conduct, and results can be ascertained rapidly.

Realizing the immense potentiality of the test and the laboratories all around the world have standardized a CP-based dot-ELISA for the early diagnosis of fasciolosis in human and ruminants with high level of sensitivity/specificity $(\sim 100 \%)$ in experimental conditions while a little bit compromised sensitivity/specificity in field conditions and recommended to use it in the field situations $[16,17,25]$. The $28 \mathrm{kDa}$ $\mathrm{CP}$ observed high-level of sensitivity in the ELISA for the detection of experimental $F$. gigantica infection in buffaloes by $3-4$ WPI $[12,16,17,20]$. The integrity of the selected CP antigen on SDS-PAGE confirmed that the antigen was made up of a single visible band, as reported by earlier scientists. In view of the absence of the reference test for the diagnosis of tropical fasciolosis, PM data on parasite prevalence would have been served in better way for the calculation of the sensitivity/specificity, but by knowing the fact of the vegetarian nature of the human population of the study area, there is no any recognized slaughterhouse of the large ruminants, so we were unable to calculate the sensitivity/specificity of the test and antigen in the field 


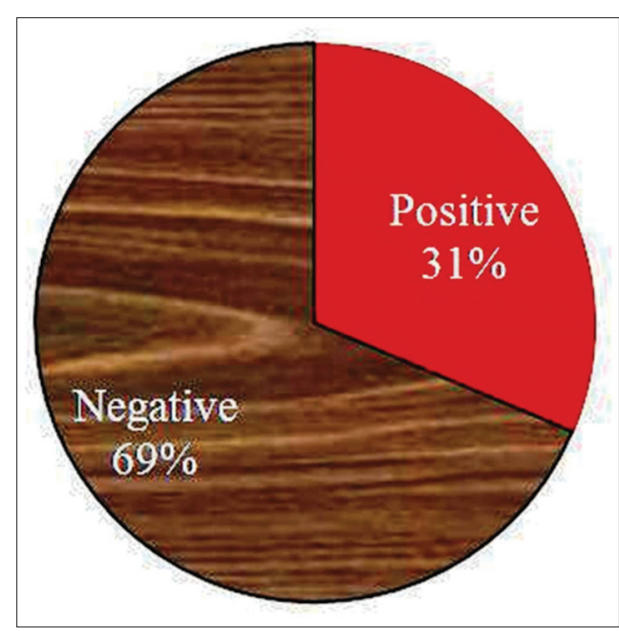

Figure-3: Percent distribution of Fasciola gigantica seropositive/negative bovines.

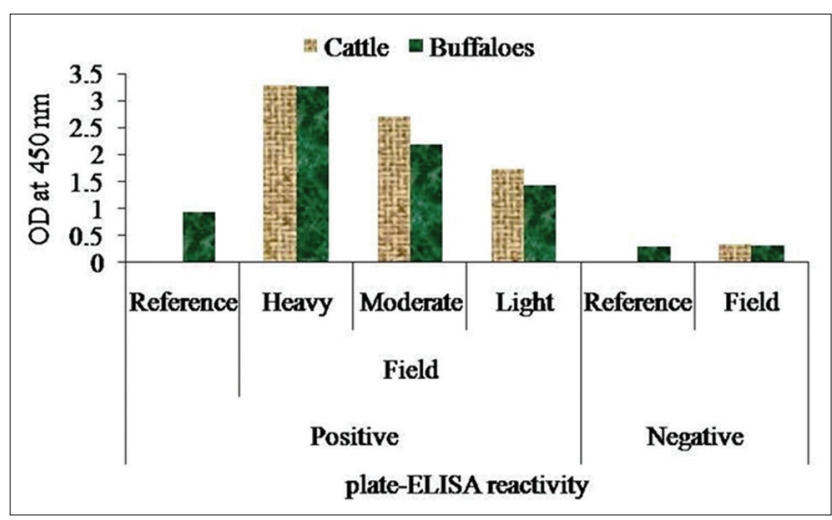

Figure-4: Plate enzyme-linked immunosorbent assay reactivity pattern of Fasciola gigantica infection.

conditions. On the other hand, the trematodes are well known for its intermittent egg shedding behavior, and further, there is no any direct correlation between the egg per gram of feces and actual parasitic load, so the use of coprological data for evaluating serological test is worthless [26]. Interestingly, Raina et al. [20] correctly pointed out that the sensitivity of the serological assay was determined by the antibody response elicited by the individual host rather the fluke burden. Hence, it is the demand of the time to describe a reference/gold standard test for the calculation of sensitivity/specificity of the new upcoming serological/molecular test for the detection of $F$. gigantica infection.

Overlapping antigens in the trematodes frequently observed cross-reactivity among themselves [13]; fortunately, in the present study, no cross-reaction was noted with the rumen amphistome, G. crumenifer, and few more researcher observed no cross-reaction with Paramphistomum epiclitum, Gigantocotyle explanatum, hydatid cyst, and Strongyloides papilossus $[14,15,20]$.

The present study recorded moderate level of prevalence (31.33\%) (Figures-1-3) of F. gigantica seropositive animal, which seems to be due to heavy rainfall resulting into plenty of water body harboring the snail intermediate host. Depending on the agroclimatic conditions and the type of the ruminant host, the researcher all over the world time to time reported the low-to-high level of prevalence of $F$. gigantica using specific diagnostic antigen in the ELISA $[10,12,18,27,28]$. The water-loving nature of the buffaloes could be hypothesized that they can have more incidence rate of $F$. gigantica over the cattle. Interestingly, in the current work, there was non-significant $(\mathrm{p}>0.05)$ difference in the seroprevalence rate of tropical liver fluke between cattle and buffaloes (Figure-1), and this might be due to the adoption of the same pattern of rearing of the large ruminants by the farmers in the study area [29].

The reactivity observed in plate-ELISA in terms of OD values at $450 \mathrm{~nm}$ between the positive and reference/field negative sera in both cattle and buffaloes was significantly $(\mathrm{p}<0.05)$ different (Figure-4). The moderately reacted bovine in dot-/plate-ELISA fall at the borderline and can be shifted toward the up-/downside of the reactivity of $F$. gigantica infection $[14,15]$.

In spite of many merits of CP-based ELISA, the one major inherited demerit is that being the antibody detection test, unable to differentiate the active and passive infection status of the animals. Hence, we have to consider the clinical status of the animals to ascertain the final infection status of the animals $[30,31]$.

\section{Conclusion}

Overall, we pass our recommendation to the said laboratory to move forward and come up with a first field-based detection kit for the tropical fasciolosis using the CP antigen in paper-/dot-ELISA, which will be very useful for field veterinarians for quick and timely detection of the animals requiring urgent therapy with the flukicide at the farmer's doorstep.

\section{Authors' Contributions}

NK planned and accomplished the overall research work. AV collected the sera and did the ELISA. NK did the data analysis and drafted the manuscript. JBS revised the manuscript. All authors read and approved the final manuscript.

\section{Acknowledgments}

The authors are thankful to Dr. O. K. Raina (Principal Scientist, IVRI, Izatnagar), for providing antigen and experimental sera to conduct seroprevalence study. We are also thankful to the Principal, Veterinary College, Navsari Agricultural University, Navsari, for providing necessary facilities and fund (plan budget head no. 12404) to complete the research work.

\section{Competing Interests}

The authors declare that they have no competing interests.

\section{References}

1. Gupta, S.C., Ghosh, S., Raina, O.K., Joseph, D., Preeti, R., Singh, B.P., Mishra, A.K., Chandra, D. and Samanta, S. 
(2008) Status and prevalence of fasciolosis in cattle and buffaloes in different agro-climatic zones of Uttar Pradesh. J. Vet. Parasitol., 22: 59-63.

2. Pandya, S.S., Hasnani, J.J., Patel, P.V., Chauhan, V.D., Hirani, N.D., Shukla, R. and Dhamsaniya, H.B. (2015) Study on prevalence of fasciolosis in buffaloes at Anand and Ahmedabad districts, Gujarat, India. Vet. World, 8(7): 870-874.

3. Elelu, N., Ambali, A., Coles, G.C. and Eisler, M.C. (2016) Cross-sectional study of Fasciola gigantica and other trematode infections of cattle in Edu local government area, Kwara state, North-Central Nigeria. Parasit. Vectors, 9: 470 .

4. Mulcahy, G. and Dalton, J.P. (2001) Cathepsin-L proteinases as vaccines against infection with Fasciola hepatica (liver fluke) in ruminants. Res. Vet. Sci., 70: 83-86.

5. WAAVP Congress. (2005) Fasciola hepatica: Suppression of Host Immune Responses and Susceptibility of Other Diseases, Annual Convention of the World Association for the Advancement of Veterinary Parasitology. p21-22.

6. Sariözkan, S. and Yalçin, C. (2011) Estimating the total cost of bovine fasciolosis in Turkey. Ann. Trop. Med. Parasitol., 105: 439-444.

7. Jean-Richard, V., Crump, L., Abicho, A.A., Naré, N.B., Greter, H., Hattendorf, J., Schelling, E. and Zinsstag, J. (2014) Prevalence of Fasciola gigantica infection in slaughtered animals in south-eastern Lake Chad area in relation to husbandry practices and seasonal water levels. BMC Vet. Res., 10: 81.

8. Fairweather, I. (2005) Triclabendazole: New skills to unravel an old(ish) enigma. J. Helminthol., 79: 227-234.

9. Gupta, S.C. and Yadav, S.C. (1992) Sexual maturity of Fasciola gigantica in experimentally infected rabbits, goats and buffaloes. Indian J. Parasitol., 16: 133-134.

10. Rehman, T., Khan, M.N., Abbas, R.Z., Babar, W., Sikandar, A. and Zaman, M.A. (2016) Serological and coprological analyses for the diagnosis of Fasciola gigantica infections in bovine hosts from Sargodha, Pakistan. $J$. Helminthol., 90(4): 494-502.

11. Valero, M.A., Bargues, M.D., Khoubbane, M., Artigas, P., Quesada, C., Berinde, L., Ubeira, F.M., Mezo, M., Hernandez, J.L., Agramunt, V.H. and Mas-Coma, S. (2016) Higher physiopathogenicity by Fasciola gigantica than by the genetically close $F$. hepatica: Experimental long-term follow-up of biochemical markers. Trans R. Soc. Trop. Med. Hyg., 110(1): 55-66.

12. Varghese, A., Raina, O.K., Nagar, G., Garg, R., Banerjee, P.S., Maharana, B.R. and Kollannur, J.D. (2012) Development of cathepsin-L cysteine proteinase based dotenzyme-linked immunosorbent assay for the diagnosis of Fasciola gigantica infection in buffaloes. Vet. Parasitol., 183: 382-385.

13. Yokananth, S., Ghosh, S., Gupta, S.C., Suresh, M.G. and Saravanan, D. (2005) Characterization of specific and cross-reacting antigens of Fasciola gigantica by immunoblotting. Parasitol. Res., 97: 41-48.

14. Kumar, N., Ghosh, S. and Gupta, S.C. (2008) Detection of Fasciola gigantica infection in buffaloes by enzyme-linked immunosorbent assay. Parasitol. Res., 104: 155-161.

15. Kumar, N., Ghosh, S. and Gupta, S.C. (2008) Early detection of Fasciola gigantica infection in buffaloes by enzymelinked immunosorbent assay and dot enzyme-linked immunosorbent assay. Parasitol. Res., 103: 141-150.

16. Yadav, S.C., Saina, M., Raina, O.K., Nambi, P.A., Jadav, K. and Sriveny, D. (2005) Fasciola gigantica cathepsin-L cysteine proteinase in the detection of early experimental fasciolosis in ruminants. Parasitol. Res., 97: 527-534.

17. Sriveny, D., Raina, O.K., Yadav, S.C., Chandra, D., Jayraw, A.K., Singh, M., Velusamy, R. and Singh, B.P. (2006) Cathepsin-L cysteine proteinase in the diagnosis of bovine Fasciola gigantica infection. Vet. Parasitol., 135: 25-31.

18. Avcioglu, H., Guven, E., Balkaya, I., Kaynar, O. and Hayirli, A. (2014) Evaluation of coprological and serological techniques for diagnosis of bovine fasciolosis. Isr. J. Vet. Med., 69(4): 203-210.

19. Yadav, P. and Singh, R. (2011) A review on anthelmintic drugs and their future scope. Int. J. Pharm. Pharm. Sci., 3(3): 17-21.

20. Raina, O.K., Yadav, S.C., Sriveny, D. and Gupta, S.C. (2006) Immuno-diagnosis of bubaline fasciolosis with Fasciola gigantica cathepsin-L and recombinant cathepsin L 1-D protease. Acta Trop., 98: 145-151.

21. Ouchterlony, O. (1964) Gel diffusion technique. In: Ackroyd, J.F., editor. Immunological Methods. Blackwell Scientific Publication, Oxford. p55-78.

22. Doyle, J.J. (1973) Skin hypersensitivity reaction induced in calves by experimental infections with Fasciola hepatica. Int. Arch. Allergy, 45: 752-758.

23. Pappas, M.G., Hajkowski, R. and Hockmeyer, W.T. (1983) Dot-enzyme linked immunosorbent assay (dot-ELISA): A micro technique for the rapid diagnosis of visceral leishmaniasis. J. Immunol. Methods, 64: 205-214.

24. Maisonnave, J. (1999) Standardization of dot immuno-peroxidase assay for field diagnosis of Fasciola hepatica infected cattle. Vet. Parasitol., 85: 259-268.

25. Cornelissen, J.B.W., Gassenbeek, C.P.H., Borgsteede, F.H.M., Holland, W.G., Harmsen, M.M. and Bocrsma, W.J.A. (2001) Early immune-diagnosis of fasciolosis in ruminants using recombinant Fasciola hepatica cathepsin-L-like protease. Int. J. Parasitol., 31: 728-737.

26. Fagbemi, B.O. and Guobadia, E.E. (1995) Immunodiagnosis of fasciolosis in ruminants using a $28 \mathrm{kDa}$ cysteine protease of Fasciola gigantica adult worms. Vet. Parasitol., 57: 309-318.

27. Damwesh, S.D. and Ardo, M.B. (2013) Detection of Fasciola gigantica antibodies using pourquier ELISA kit. Sokoto J. Vet. Sci., 11(2): 43-48.

28. Chamuah, J.K., Jacob, S.S., Sakhrie, A. and Borkotoky, D. (2014) Serological prevalence of Fasciola gigantica in mithun (Bos frontalis). Int. J. Livest. Res., 4(5): 15-20.

29. Sabapara, G.P., Desai, P.M., Kharadi, V.B., Saiyed, L.H. and Singh, R.R. (2010) Housing and feeding management practices of dairy animals in the tribal area of South Gujarat. Indian J. Anim. Sci., 80: 1022-1027.

30. Palmer, D.G., Lyon, J., Palmer, M.A. and Forshaw, D. (2014) Evaluation of a copro-antigen ELISA to detect Fasciola hepatica infection in sheep, cattle and horses. Aust. Vet. J., 92(9): 357-361.

31. Abdolahi-Khabisi, S. and Sarkari, B. (2016) Detection of Fasciola hepatica and Fasciola gigantica common and uncommon antigens, using rabbit hyper immune serum raised against their excretory-secretory and somatic antigens. J. Parasit. Dis., 40(4): 1552-1557. 